\title{
Females Experience a More Severe Disease Course in Batten Disease
}

\author{
Jennifer Cialone, Heather Adams, Erika F. Augustine, Frederick J. Marshall, Jennifer M. \\ Kwon, Nicole Newhouse, Amy Vierhile, Erika Levy, Leon S. Dure, Katherine R. Rose, Denia \\ Ramirez-Montealegre, Elisabeth A. de Blieck, and Jonathan W. Mink \\ University of Rochester
}

\begin{abstract}
Juvenile neuronal ceroid lipofuscinosis (JNCL; CLN3 disease; Batten disease) is an autosomal recessive neurodegenerative disease of childhood. Symptoms typically present at school age with vision loss followed by progressive cognitive decline, motor dysfunction, seizures, and behavior problems. Studies on sex differences in JNCL have yielded mixed results, but parent anecdotes suggest that females experience a more precipitous disease course. Therefore, we sought to determine if sex-based differences exist in JNCL. We used data from the Unified Batten Disease Rating Scale (UBDRS), the Batten Disease Support and Research Association (BDSRA) database, and the PedsQL quality of life (QoL) survey to evaluate sex-based differences in functional independence and time from symptom onset to death. On average, females had JNCL symptom onset one year later and death one year earlier than did males. Despite a later age at onset, females had lower functional capability, earlier loss of independent function, and lower physical QoL. Future research in sex differences in JNCL may help to further understand the biological mechanisms underpinning the disease course and may point to targeted therapies.
\end{abstract}

\section{Introduction}

The neuronal ceroid lipofuscinoses (NCLs) are a heterogeneous group of inherited lysosomal storage diseases that constitute the most prevalent neurodegenerative diseases of childhood (Santavuori et al 2000). The juvenile type is the most common NCL. It is inherited in an autosomal recessive manner and is characterized by vision loss, seizures, dementia, behavioral difficulties, and motor impairment. Symptoms begin in school-age children, and the disease progresses to death, usually during the third decade of life (Boustany 1996; Goebel and Kohlschütter 2001; Hofman et al 1999).

Disease features and overall clinical course of JNCL have not provided obvious evidence for sex differences. However, some differences have been suggested and attributed to hormonal factors. Compared to healthy female controls, JNCL females more commonly have acne, hirsutism, and hyperandrogenemia that are not solely attributable to medication side effects. JNCL females may also have an earlier age at menarche (Aberg et al 2002). Studies of sexbased differences in the severity of JNCL-specific behavioral, cognitive, and physical symptoms have yielded mixed results. In one study, JNCL females demonstrated more psychiatric problems based on the Child Behavior Checklist (CBCL), specifically in the internalizing, anxiety/depression, and attention domains. Females were also more likely to be treated with psychotropic medications (Bäckman et al 2005). However, another study using the CBCL found no sex differences (Adams et al 2010). Cognitive testing has not 
revealed a significant difference between males and females (Adams et al 2007). Finally, prior studies have shown no significant sex differences in overall physical impairment (Adams et al 2010) or rate of physical decline (Kwon et al 2011).

Despite the lack of consistent evidence for sex differences in JNCL, several parents have related anecdotes suggesting that females experience a more precipitous decline than do males. Therefore, we sought to determine if there are sex-based differences in aspects of JNCL that affect functional independence and time from symptom onset to death.

\section{Methods}

\section{The Unified Batten Disease Rating Scale (UBDRS)}

We developed the Unified Batten Disease Rating Scale (UBDRS) to quantify disease progression in JNCL. The UBDRS has been shown to be a valid and reliable clinical rating instrument for JNCL (Kwon et al 2011; Marshall et al 2005). The UBDRS encompasses four quantitative subscales: Physical Impairment, Behavior, Seizures, and Functional Capability. Additionally, the UBDRS assesses the chronology of symptom onset based on parent report. Between 2002 and 2010, we administered the UBDRS to nearly $100 \mathrm{JNCL}$ subjects to characterize the natural history of the disease and to quantify the rate of progression (Kwon et al 2011; Marshall et al 2005).

UBDRS data were obtained at annual meetings of the Batten Disease Support and Research Association (BDSRA) and at the University of Rochester Batten Center (URBC). Unless otherwise noted, all analyses were performed on data from subjects in our cohort. These subjects had been clinically diagnosed with JNCL and were genetically confirmed to have CLN3 mutations (Rothberg et al 2004). Some subjects did not complete all sections of the UBDRS at each assessment; therefore, the number of subjects varied slightly for each anaylsis. The study was approved by the University of Rochester Research Subjects Review Board; parents provided informed consent for their child's participation.

To evaluate disease progression, we analyzed data from the Physical Impairment and Functional Capability subscales of the UBDRS. The Physical Impairment subscale is scored based on direct physical examination of the following items, each rated from 0 (normal) to 4 (severe): speech clarity, abnormal repetitive speech sounds, tongue protrusion, visual acuity, neck and extremity tone, extremity strength, rapid alternating movements in hands and feet, upper extremity dystonia, normal spontaneous movements, gait, retropulsion, motor tics or stereotypies, myoclonus, tremor, dysmetria, and appendicular chorea. The Functional Capability subscale is based on a parent interview and evaluates school performance, ability to complete chores, ability to play games, participation in activities of daily living (ADLs), and overall level of care needed. Parents reported their child's level of function in each area based on the child's current vision status. They then reported assumed ability if the child had full vision, in attempt to remove the effect of blindness on functional capability. Items were scored from 0 (most impaired) to 3 (normal). These scores were combined to reflect total capability, with lower scores indicating worse function.

\section{Age at Symptom Onset Analysis}

At each study visit, we asked parents to recall the age at onset of specific JNCL symptoms including vision loss, behavior problems, cognitive decline, motor impairment, seizures, sleeping difficulty, and feeding difficulty. To obtain a best estimate of age at first symptom onset, we limited this analysis to subjects whose parents reported vision loss as the first symptom. Vision loss is the most common first symptom that is most clearly linked to JNCL disease (Marshall et al 2005). Some subjects with multiple assessments had slight variation 
in the estimated age at onset from year to year. For those subjects, we used an average of the reported ages.

To determine the average age at which other symptoms began, the estimated age at onset of each JNCL symptom was determined from the most recent JNCL history assessment. The mean age at onset across subjects was determined for each symptom.

For the age at onset analyses, standard parametric statistics were used. Differences between males and females were determined with t-tests.

\section{Clinical Outcomes Assessments}

The UBDRS Physical Impairment subscale quantifies a variety of physical aspects of JNCL disease. Speech and gait are two items in the subscale that strongly impact independent function. We determined the age at which subjects lost the ability to ambulate independently and the age at which speech became unintelligible. Using the Functional Capability subscale, we determined the age at which subjects were no longer able to perform independent ADLs. Kaplan Meier survival curves were constructed for each variable. The age at which subjects lost the ability to discern objects served as a control since vision loss is often an early symptom in JNCL. We assessed scores from all UBDRS assessments completed for the subjects over the years, so each subject may have had multiple scores included in this analysis. A logrank test was performed to compare survival curves between males and females (MedCalc v11.4.4). One subject had an abnormal disease course not consistent with a classic JNCL phenotype and was excluded from this analysis.

\section{Quality of Life Assessments}

We assessed quality of life (QoL) in JNCL subjects using the Pediatric Quality of Life questionnaire (Varni et al 2001). Only subjects who had confirmation of CLN3 mutations and whose parents had completed at least one questionnaire were included. This survey measures health-related QoL over the previous month in four domains: Physical Functioning, Emotional functioning, School Functioning, and Social Functioning. Each item is rated from 0 (never a problem) to 4 (almost always a problem). The Physical Functioning domain assesses problems with walking, running, sports participation, heavy lifting, bathing, chores, hurts or aches, and energy level. The Emotional Functioning domain assesses feelings of fear, sadness, or anger; difficulty sleeping; and worries about what will happen to him or her. The School Functioning domain assesses problems with paying attention in class, forgetting things, keeping up with schoolwork, and missing school (either for feeling poorly, having a doctor's appointment, or being hospitalized). The Social Functioning domain ability to get along with peers, peers wanting to be friends with the child, problems with teasing by peers, ability to do things that other peers can do, and keeping up with peers. Items were reverse scored and linearly transformed to a 0-100 score so that higher values represented higher health-related QoL. The mean score for each domain was calculated, and this value was used for each domain's summary score. These questionnaires were sent out to families biannually, and scores from the most recently completed questionnaire were used in this analysis. $\mathrm{T}$ tests were performed to compare male and female summary scores for each domain.

\section{Age at Death Analysis}

Due to the small number of deceased subjects, we could not meaningfully assess age at death in our cohort. Instead, we obtained data from a de-identified database provided by the BDSRA, which contains information on year of birth, NCL type, and age at death of individuals affected with Neuronal Ceroid Lipofuscinoses (NCLs). Subjects identified in the database as having clinical JNCL were included. Genetic confirmation information was not 
available. We excluded subjects with an age at death greater than 30 years since death usually occurs during the third decade of life in classic JNCL. Thus, an older age at death raised doubts about the diagnosis of JNCL. The mean age at death for males and females was determined and a Mann-Whitney U test was performed.

\section{Statistical Analyses}

All statistical analyses were performed using Statistica ver 6.1 unless otherwise noted.

\section{Results}

\section{Age at symptom onset}

Data on age at symptom onset were available for 83 subjects (39 female, 44male) with confirmed mutations in CLN3. Parents of 60 subjects ( 30 female, 30 male) reported vision loss as their child's first symptom of JNCL. The mean age at disease onset as marked by vision loss was one year later in females $(6.2+/-1.4$ years $)$ than in males $(5.2+/-1.0$ years) $(\mathrm{p}=0.003)$ (Figure 1). Twenty-three subjects had an initial symptom that was not vision loss. When these were added to the sample, the mean age at symptom onset was slightly younger for both females $(5.7+/-1.7$ years) and males $(4.6+/-1.5)$, but the difference remained similar and significant $(\mathrm{p}=0.002)$. Parents of the remaining subjects reported behavior difficulties $(\mathrm{N}=11)$, cognitive decline $(\mathrm{N}=6)$, sleep disturbances $(\mathrm{N}=3)$, seizures $(\mathrm{N}=1)$, feeding difficulties $(\mathrm{N}=1)$, and other $(\mathrm{N}=1)$ as initial symptoms.

Similar to vision loss, behavioral symptoms also began later in females (Table 1). There was no difference in mean age at onset of seizures, cognitive impairment, or motor symptoms. Vision loss was the first symptom in females and males, but the order of other symptom onset was slightly different due to the earlier age at onset for behavioral symptoms in males. In JNCL females, the average order of symptom onset was vision loss, cognitive impairment, seizures, behavioral symptoms, and motor symptoms (Figure 2A). In JNCL males, the average order of symptom onset was vision loss, behavioral symptoms, cognitive impairment, seizures, and motor symptoms (Figure 2B).

\section{Clinical Outcomes}

Thirty-seven females and 41 males completed the UBDRS Functional Capability subscale. As expected, scores decreased with increasing age, reflecting greater disease severity over time (Figure 3). Both males and females decreased an average of 1.2 points per year of age on this subscale. Although the slopes were parallel, there was a slight offset such that on average, females scored one point lower than males of the same age. On average, females also reached comparable levels of disability one year earlier than males.

Two hundred nineteen UBDRS Physical Impairment subscale evaluations were performed for 83 subjects. These evaluations (92 for female subjects and 127 for male subjects) were used to assess age at complete loss of independent gait, intelligible speech, and vision. Two hundred seventeen UBDRS Functional Capability evaluations were performed ( 87 for female subjects, 130 for male subjects) to determine age at loss of independent ADLs. Early in the disease course, the great majority of both females and males were able to walk independently, speak intelligibly, and perform independent ADLs with no apparent sex difference. Approximately 10-15 years after disease onset, a difference between females and males emerged such that females lost independence in ADLs earlier than males. The difference in survival curves was significant $(\mathrm{p}=0.005)$. Median survival time for independent ADLs was 12 years for females and 13 years for males (Figure 4). There was also a one-year difference between females (14 years) and males (15 years) in median survival time for both independent gait and intelligible speech. However, the difference 
between survival curves was not significant. Both males and females had a median survival time of 10 years for complete vision loss.

\section{Quality of Life}

Parents of 49 subjects ( 22 females, 27 males) completed the PedsQL questionnaire. The mean age at time of completion was similar for females ( $16.3+/-4.4$ years) and males (14.4 $+/-6.1$ years) $(\mathrm{p}=0.23)$. Some parents did not complete all sections (social $\mathrm{N}=1$, school $\mathrm{N}=4$ ). Scores ranged from 0 to 100, with higher scores denoting a better QoL. In the physical domain, females demonstrated poorer QoL (Figure 5). Social, emotional, and school QoL domains were not different between males and females (Table 2).

\section{Age at death}

There were 226 subjects (128 females, 98 males) with clinically diagnosed JNCL who died at or before the age of thirty years included in this analysis. Age at death was $20.9+/-4.5$ years for females and $22.2+/-4.2$ years for males $(\mathrm{p}<0.03$ ) (Figure 6$)$. If all subjects $(\mathrm{n}=$ $254)$ were included, regardless of age at death, females died on average one year earlier than males $(\mathrm{p}<0.05)$.

\section{Discussion}

Females with JNCL experienced a later onset but more rapidly progressive disease course than did males. On average, females experienced JNCL symptom onset one year later and death one year earlier. Females also experienced earlier loss of independence in ability to perform ADLs and a tendency to earlier loss of independent gait and intelligible speech. The ability to perform ADLs is influenced by multiple domains affected in JNCL children, including cognition, physical ability, and vision. Thus, independence in ADLs is a good general indicator of overall disease severity. In addition, parents reported a poorer physical QoL in female children. Other QoL domains were not different between males and females, suggesting that physical factors contribute more greatly to loss of independence than do cognitive or behavioral factors. However, our results show that sex differences are not limited to physical impairment but include age at onset of vision loss and behavioral symptoms.

A biological basis for the more severe disease course in females is not obvious. JNCL is an autosomal recessive disease that affects males and females at a similar rate. In adult-onset neurodegenerative disease, female sex is often associated with a milder course. In tests of fine motor control and speech articulation in subjects with Parkinson's disease (PD), women outperform men (Gillies and McArthur 2010). The protective effect of female sex may be due to hormonal factors. Indeed, decreasing levels of endogenous estradiol in women is associated with symptom worsening in PD (Gillies and McArthur 2010). Estrogen may also have a protective role in neurodegenerative diseases (Brann et al 2007; McEwen and Alves 1999). Despite this proposed protective benefit of estrogen, JNCL females demonstrated earlier loss of independent function during post-pubertal years, when estrogen levels should be elevated. It is possible that, rather than being neuroprotective, estrogen plays a role in the more rapid course in JNCL females. Additionally, the second X-chromosome in females may contribute to sex differences in JNCL. For example, due to imprinting, females may have deleterious effects from a paternally inherited X-chromosome gene. Alternatively, there may be some protective gene on the Y-chromosome.

One explanation for the sex-based differences in JNCL may relate to the proposed autoimmune component to its pathogenesis. Multiple autoantibodies have been detected in the CNS of both animal models and human JNCL (Chattopadhyay et al 2002; Lim et al 
2006). Suppressing the immune system reduces these autoantibodies and slows disease progression in animal models (Seehafer et al 2011). Females have a higher incidence of autoimmune diseases in general, but sex-based differences in the clinical phenotypes of these diseases vary (McCombe et al 2009). Differences in incidence and clinical course may be partially attributed to the sexual dimorphism observed in the human immune system. Females have a more robust antibody response to vaccines regardless of menstrual status, and estrogen itself has been shown to increase antibody production. Diseases with a predominant autoantibody immune process, such as Systemic Lupus Erythematosus (SLE), are more likely to worsen with higher estrogen levels (Grimaldi 2006). Therefore, while estrogen may be neuroprotective, it may also increase autoantibody production in JNCL and thus accelerate disease progression.

Another potential contributor to earlier age at loss of independent function may be a lower baseline muscle mass in females. In the general population, males have a higher percentage of lean body mass even before the onset of puberty (Rowland 2005). It is possible that, because males start with greater muscle mass, the degenerative effects on physical function are seen later in JNCL boys. However, it is not clear if loss of functional independence is related to muscle mass or to other aspects of motor control including cognitive planning. Thus, while muscle mass may contribute to preserved physical function in males, it is unlikely to account entirely for these differences.

The discrepancy in independent function may also be due to social constructs of gender expectations. Parents demonstrate a gender bias in physical expectations for children starting at a young age. Although infants do not have sex-based differences in motor development, mothers of female infants have lower expectations for their children's ability to perform motor tasks (Mondschein et al 2000). After infancy, parents allow boys more independence with less supervision during risky behaviors. In physical tasks that both girls and boys are able to complete, parents are still more likely to assist their daughters while encouraging independence from their sons (Morrongiello and Dawber 1999). Translating these results to $\mathrm{JNCL}$, it is possible that parents of daughters set lower expectations for physical ability. They may be more likely to assist with ambulation and ADLs and less likely to encourage independent function. It is also possible that gender bias contributes to the reported later age at onset of vision loss in females. Thus, the observed differences in function and age at onset may result in part from differences in parental expectations and behavior.

There are several potential limitations to our study. We relied on parent recall of symptom onset, which created a potential recall bias. There is some variability from year to year in reported age at onset, which could also reflect differences in the way that questions were asked. In an effort to minimize these limitations, we only analyzed the data obtained at the most recent assessment. Another limitation is that the age at death analysis included subjects who did not have genetic confirmation data available. Thus, it is possible that some subjects did not have mutations in $C L N 3$. We attempted to eliminate incorrectly diagnosed subjects by excluding those with an age at death uncharacteristically late for classic JNCL disease (greater than 30 years of age). This age criterion was based on our extensive clinical experience with JNCL disease. However, if all subjects were included, regardless of age at death, comparable results were obtained.

Despite these potential limitations, the data indicating later age at onset and more rapid progression in females are compelling. Furthermore, we have no reason to think that the study design introduced a systematic bias for sex-based differences. It is possible that a combination of several factors, including autoimmunity, muscle mass differences, parental expectations, or others result in a more rapid disease progression in females. The biological mechanism underlying neurodegeneration in JNCL remains unknown. It is possible that 
identification of the primary mechanism will provide an explanation for some of these differences. Additional work on specific sex-based differences in JNCL may provide a better understanding of the molecular underpinnings of the disease process and point the way to future therapeutics.

\section{Acknowledgments}

The authors would like to thank Paul Rothberg, $\mathrm{PhD}$ for his contributions to genetic data. We also thank the patients and families. The project described was supported by Award Number U54NS065768 from the National Institute Of Neurological Disorders And Stroke. The content is solely the responsibility of the authors and does not necessarily represent the official views of the National Institute Of Neurological Disorders And Stroke or the National Institutes of Health." This work was also supported by NIH Grants R01NS060022, K12NS066098, K23NS058756, and TL1RR024136, the Batten Disease Support and Research Association, the Strong Children's Research Center, and the Geoffrey Waasdorp Pediatric Neurology Fund.

\section{References}

Aberg LE, Tiitinen A, Åutti TH, Kivisaari L, Santavouri P. Hyperandrogenism in girls with juvenile neuronal ceroid lipofuscinosis. Eur J Paediatr Neurol. 2002; 6:199-205. [PubMed: 12374586]

Adams HR, Beck CA, Levy E, et al. Genotype does not predict severity of behavioural phenotype in juvenile neuronal ceroid lipofuscinosis (batten disease). Dev Med Child Neurol. 2010; 52:637-643. [PubMed: 20187884]

Adams HR, Kwon J, Marshall FJ, de Blieck EA, Pearce DA, Mink JW. Neuropsychological symptoms of juvenile-onset batten disease: Experiences from 2 studies. J Child Neurol. 2007; 22:621-627. [PubMed: 17690071]

Bäckman ML, Santavuori PR, Åberg LE, Aronen ET. Psychiatric symptoms of children and adolescents with juvenile neuronal ceroid lipofuscinosis. J Intellect Disabil Res. 2005; 49:25-32. [PubMed: 15634309]

Boustany, R-M. Batten disease or neuronal ceroid lipofuscinosis. In: Moser, HW., editor. Handbook of clinical neurology. Vol. 22. Elsevier Science B.V; 1996. p. 671-700.

Brann DW, Dhandapani K, Wakade C, Mahesh VB, Khan MM. Neurotrophic and neuroprotective actions of estrogen: Basic mechanisms and clinical implications. Steroids. 2007; 72:381-405. [PubMed: 17379265]

Chattopadhyay S, Ito M, Cooper JD, et al. An autoantibody inhibitory to glutamic acid decarboxylase in the neurodegenerative disorder batten disease. Hum Mol Genet. 2002; 11:1421-1431. [PubMed: 12023984]

Gillies GE, McArthur S. Estrogen actions in the brain and the basis for differential action in men and women: A case for sex-specific medicines. Pharmacol Rev. 2010; 62:155-198. [PubMed: 20392807]

Goebel HH, Kohlschütter A. Dementia in the neuronal ceroid-lipofuscinoses. Adv Exp Med Biol. 2001; 487:211-217. [PubMed: 11403161]

Grimaldi CM. Sex and systemic lupus erythematosus: The role of the sex hormones estrogen and prolactin on the regulation of autoreactive b cells. Curr Opin Rheumatol. 2006; 18:456-461. [PubMed: 16896282]

Hofman, I.; Kohlschütter, A.; Santavuori, P., et al. Cln3 juvenile ncl. In: Goebel, HH.; Mole, SE.; Lake, BD., editors. The neuronal ceroid lipofuscinoses (batten disease). IOS Press; 1999. p. 55-73.

Kwon JM, Adams H, Rothberg PG, et al. Quantifying physical decline in juvenile neuronal ceroid lipofuscinosis (batten disease). Neurology. 2011 in press.

Lim MJ, Beake J, Bible E, et al. Distinct patterns of serum immunoreactivity as evidence for multiple brain-directed autoantibodies in juvenile neuronal ceroid lipofuscinosis. Neuropathol Appl Neurobiol. 2006; 32:469-482. [PubMed: 16972881]

Marshall FJ, de Blieck EA, Mink JW, et al. A clinical rating scale for batten disease: Reliable and relevant for clinical trials. Neurology. 2005; 65:275-279. [PubMed: 16043799]

McCombe PA, Greer JM, Mackay IR. Sexual dimorphism in autoimmune disease. Curr Mol Med. 2009; 9:1058-1079. [PubMed: 19747114] 
McEwen BS, Alves SE. Estrogen actions in the central nervous system. Endocr Rev. 1999; 20:279307. [PubMed: 10368772]

Mondschein ER, Adolph KE, Tamis-LeMonda CS. Gender bias in mothers' expectations about infant crawling. J Exp Child Psychol. 2000; 77:304-316. [PubMed: 11063631]

Morrongiello BA, Dawber T. Parental influences on toddlers' injury-risk behaviors: Are sons and daughters socialized differently? Journal of Applied Developmental Psychology. 1999; 20:227251.

Rothberg PG, Ramirez-Montealegre D, Frazier SD, Pearce DA. Homogeneous polymerase chain reaction nucleobase quenching assay to detect the $1-\mathrm{kbp}$ deletion in $\operatorname{cln} 3$ that causes batten disease. J Mol Diagn. 2004; 6:260-263. [PubMed: 15269304]

Rowland, T. Children's exercise physiology. Champaign, IL: Human Kinetics; 2005. Aerobic fitness.

Santavuori P, Lauronen L, Kirveskari E, Åberg L, Sainio K, Autti T. Neuronal ceroid lipofuscinoses in childhood. Neurol Sci. 2000; 21:S35-41. [PubMed: 11073226]

Seehafer SS, Ramirez-Montealegre D, Wong AM, et al. Immunosuppression alters disease severity in juvenile batten disease mice. J Neuroimmunol. 2011; 230:169-172. [PubMed: 20937531]

Varni JW, Seid M, Kurtin PS. Pedsql 4.0: Reliability and validity of the pediatric quality of life inventory version 4.0 generic core scales in healthy and patient populations. Med Care. 2001; 39:800-812. [PubMed: 11468499] 


\section{Synopsis}

Females with Batten Disease experience a shorter disease course, earlier loss of independence, and worsened quality of life which suggests that in general, females have a more severe disease course. 


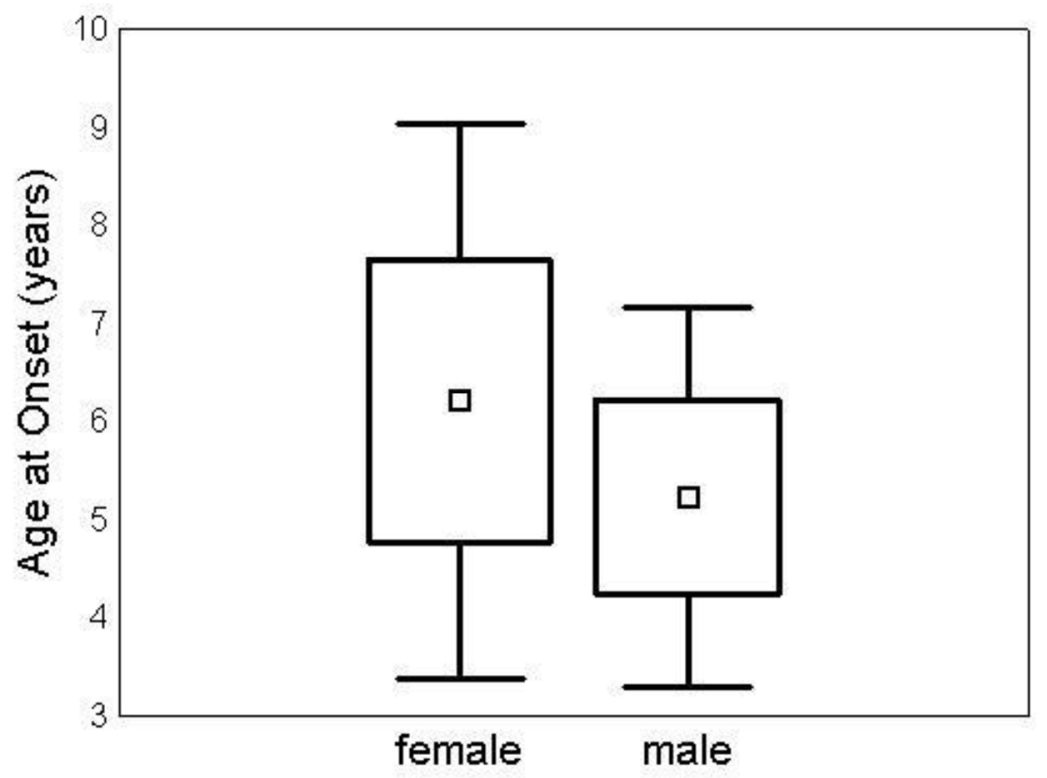

Figure 1. Disease onset was one year later in girls

Mean age at onset was $6.2+/-1.4$ years in females $(\mathrm{N}=30)$ and $5.2+/-1.0$ years in males $(\mathrm{N}=30), \mathrm{t}(60)=3.08(\mathrm{p}=0.003) . \square=$ mean. Box $=$ mean $+/-\mathrm{SD}$. Whiskers $=$ mean $+/-$ $1.96 * \mathrm{SD}$. 

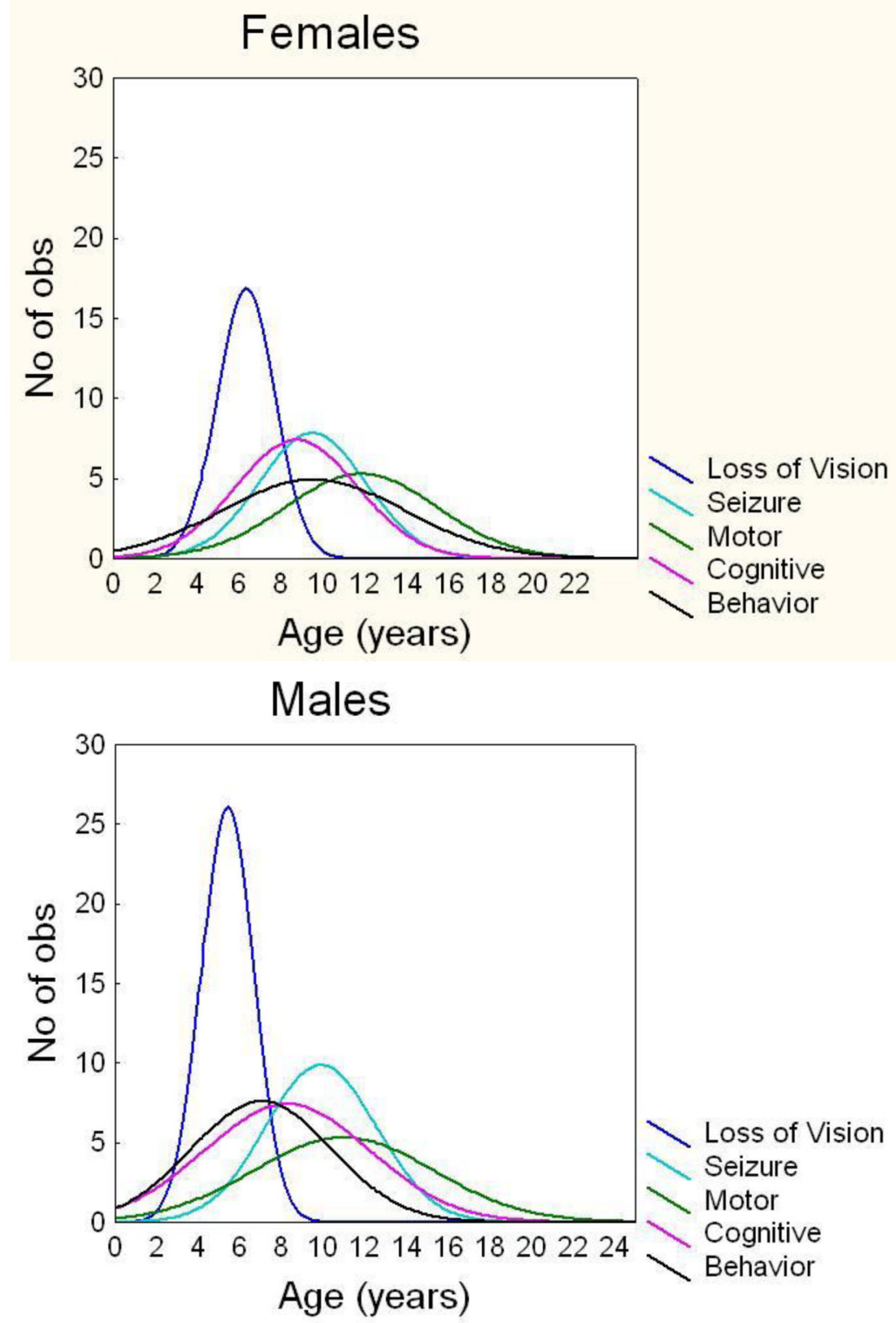

Figure 2. Females had a later age at onset of vision loss and behavior problems Histogram of the distribution of age at onset in females (A) and males (B) for vision loss, cognitive decline, seizures, behavior problems, and motor impairment. 


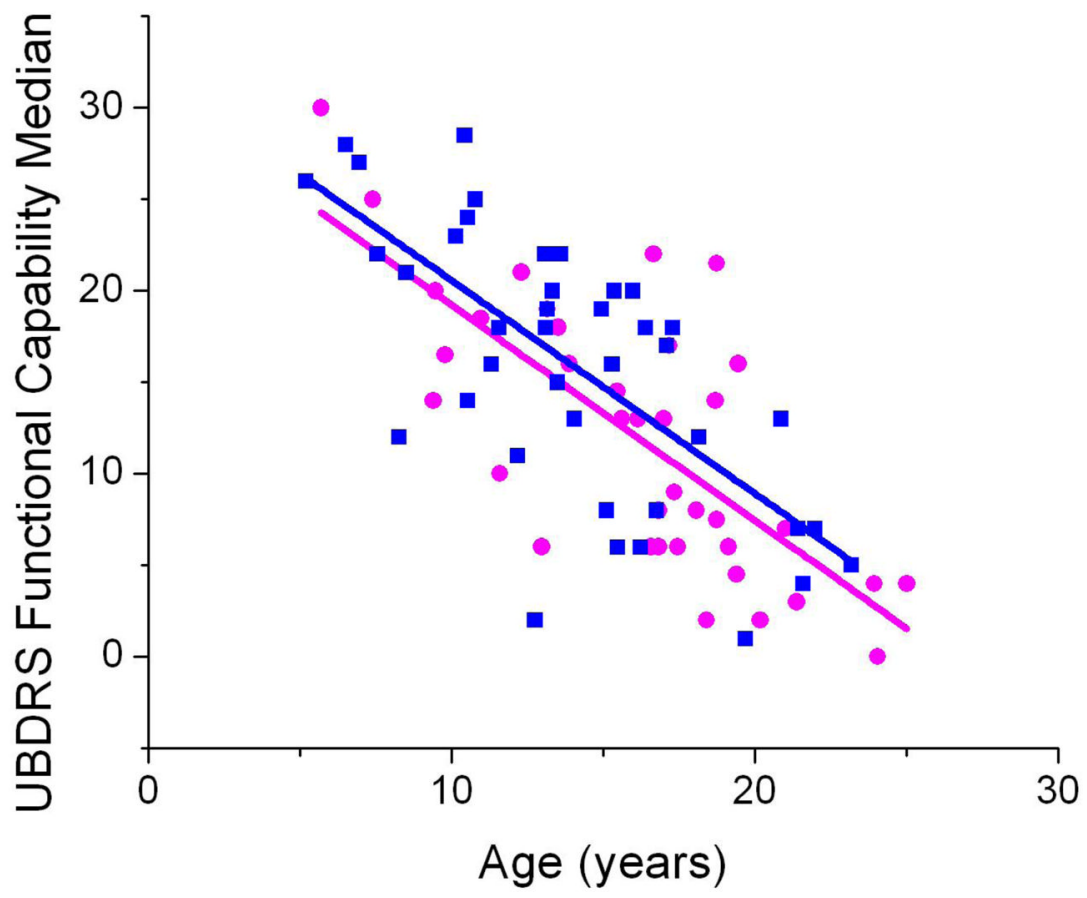

Figure 3. At all ages, females had a lower average Functional Capability score Median UBDRS Functional Capability scores were determined from subjects' most recent assessments (Female $\mathrm{N}=37$, Male $\mathrm{N}=40$ ). Lower scores reflect lower capability. Females $(\bullet)$ had overall lower scores than did males $(\boldsymbol{)})$ but there was no difference in rate of decline. Female linear fit: $y=-1.2 x+31$. Male linear fit: $y=-1.2+32$. 


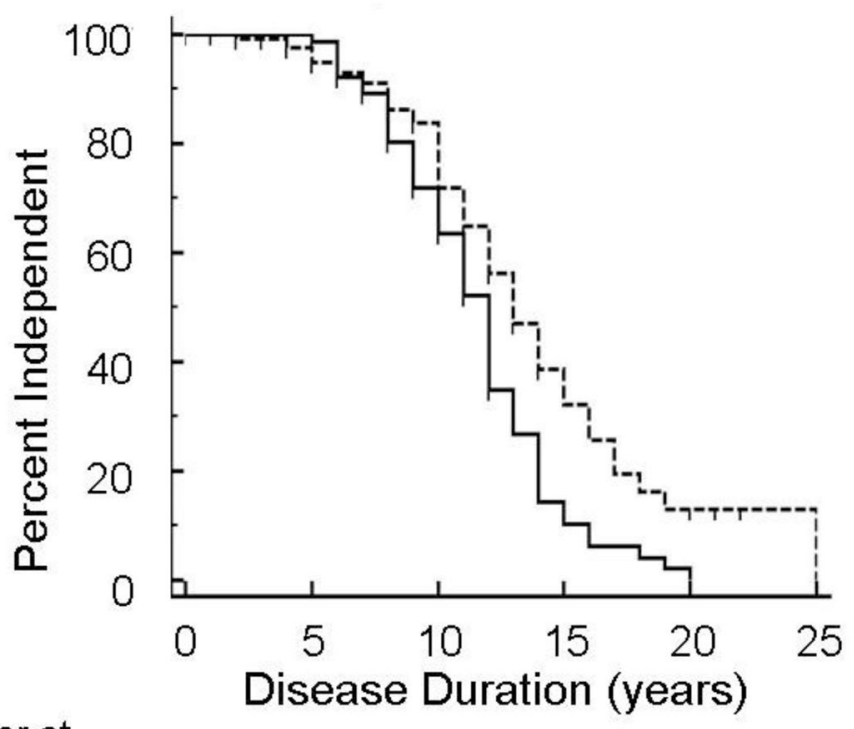

Number at

risk

Group: Female

$\begin{array}{llllll}85 & 74 & 34 & 5 & 0 & 0\end{array}$

Group: $\quad$ Male

$\begin{array}{llllll}130 & 101 & 42 & 10 & 3 & 0\end{array}$

Figure 4. Females lost independent ADLs earlier in the disease course

Kaplan-Meier survival curve of female (solid line) and male (dotted line) loss of independent ADLs, p=0.005. Females = -------- Males = - - - - 


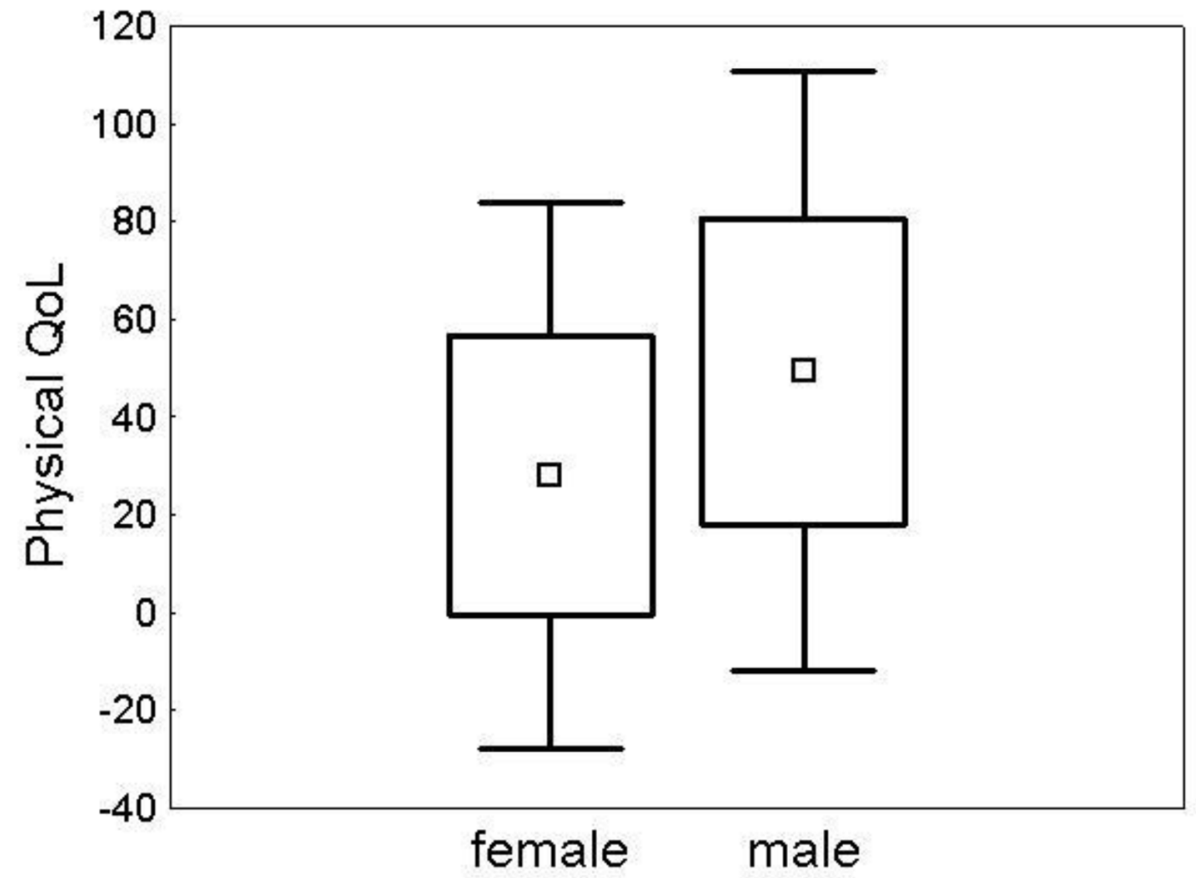

Figure 5. Females had significantly lower physical QoL

Mean physical QoL was $28.09+/-28.46$ in females $(\mathrm{N}=22)$ and $49.37+/-31.25$ in males $(\mathrm{N}=27), \mathrm{t}(47)=-2.47(\mathrm{p}=0.02) . \square=$ mean. Box $=$ mean $+/-$ SD. Whiskers $=$ mean $+/-$ $1.96 *$ SD. 


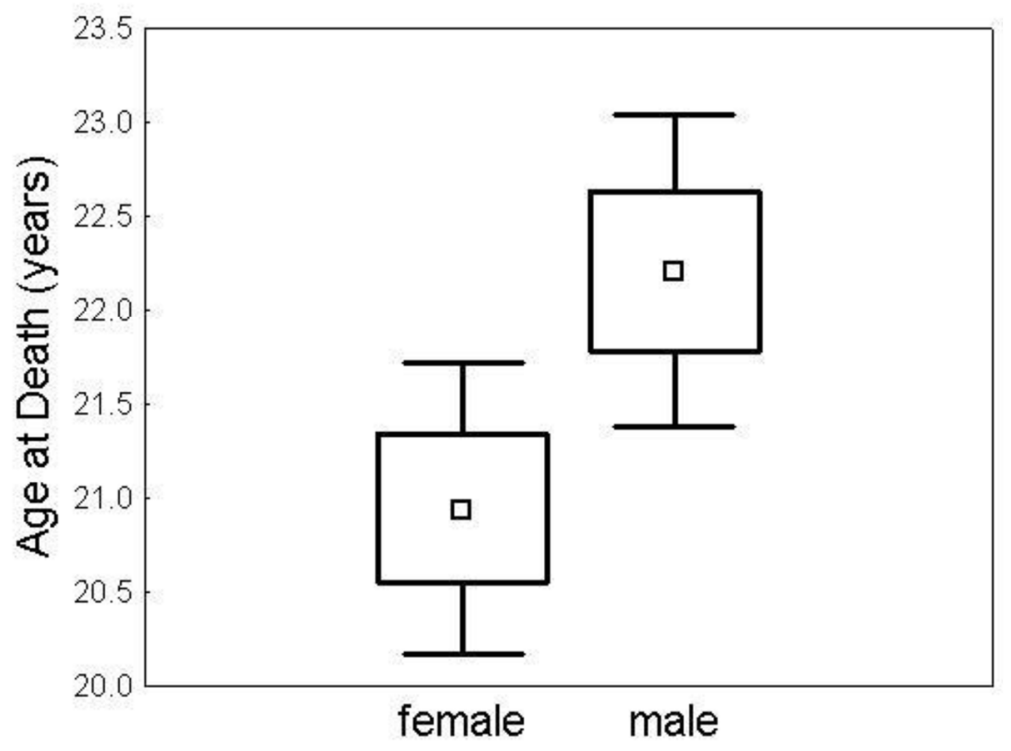

Figure 6. Females had an earlier age at death

Mean age at death was $20.95+/-4.46$ years in females $(\mathrm{N}=128)$ and $22.21+/-4.20$ years in males $(\mathrm{N}=98), \mathrm{t}(224)=-2.17(\mathrm{p}-0.03) . \square=$ mean. Box $=$ mean $+/-\mathrm{SD}$. Whiskers $=$ mean $+/-$ $1.96 * \mathrm{SD}$. 


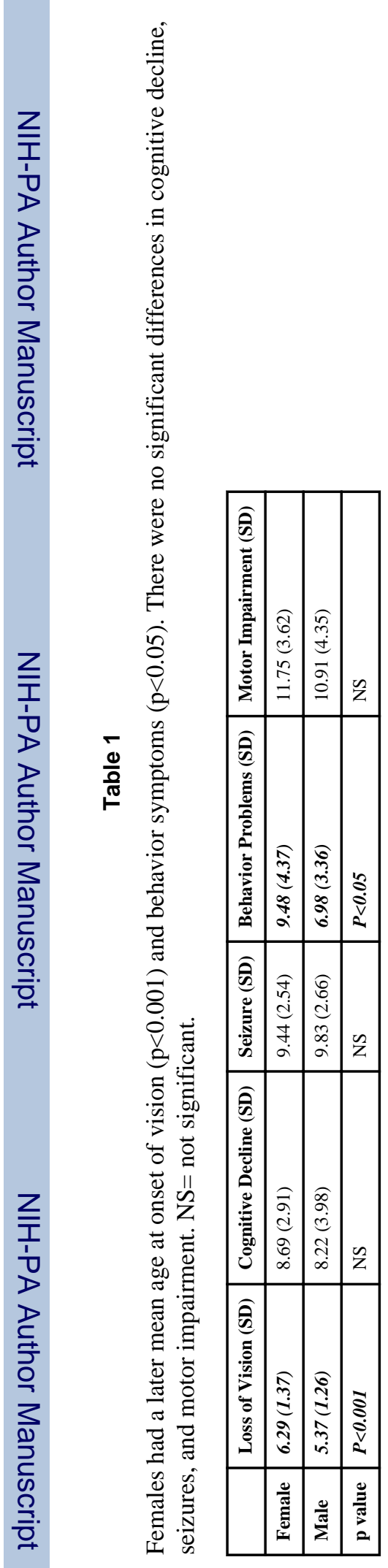

J Inherit Metab Dis. Author manuscript; available in PMC 2013 May 1. 


\section{Table 2}

Social, emotional, and school QoL were no different between males and females.

\begin{tabular}{cccc}
\hline PedsQL & Mean (SD) Females & Mean (SD) Males & $t(d f): p$ \\
\hline Social & $47.50(22.29)$ & $48.08(18.77)$ & $-0.10(46):$ NS \\
Emotional & $57.27(25.06)$ & $64.26(20.27)$ & -1.08 (47): NS \\
School & $51.90(20.77)$ & $55.63(23.79)$ & $-0.56(43):$ NS \\
\hline
\end{tabular}

